IOSR Journal of Pharmacy

ISSN: 2250-3013, www.iosrphr.org

\||| Volume 2 Issue 5 ||| Sep-Oct. 2012\|\| PP.01-03

\title{
Fetal mesenchymal stromal cells secretome regenerate skin wounds via collagen synthesis
}

\author{
*MangalaGowri.A ${ }^{\underline{a},}$, Rajasundari.M ${ }^{\underline{a}}$, Dhinakar raj ${ }^{\underline{a}}$ and A.Gnanamani ${ }^{\underline{b}}$ \\ ${ }^{a}$ Department of Animal Biotechnology, Madras Veterinary College, Chennai 600 007, India \\ ${ }^{b}$ Central Leather Research Institute, Adyar, Chennai 600025
}

\begin{abstract}
Stem cells derived from bone marrow are mesenchymal (MSC) and hematopoietic types among which MSCs are a promising source for regenerative medicine and cell based therapies). Bone marrow contains powerful mesenchymal stem cells and unanticipated is the realization that the MSCs secrete a large spectrum of bio active molecules having potential for regenerative signaling. This report analyzed the potential of pre clinical curative effect for standardized application of tissue regenerative treatment using secretome from mesenchymal stem cells by skin regeneration in situ. The necessary conditions for skin physiological repair and regeneration of wound such as physiological environment on insulted tissue through the presence of regenerative molecules and by analysis for stem cells regeneration in situ was carried out. Full-thickness skin wound can be healed physiologically and the skin tissues can be regenerated by standardized procedures and appliance of mesenchymal stem cells secretome opened up the possibilities of exploring stem cell derived proteins or products in place of stem cells for cell based therapy, a future focus in regenerative therapies.
\end{abstract}

Keywords-Fetal, mesenchymal stem cell, secretome, wound, in situ, fibrous, replacement

\section{INTRODUCTION}

The theory of Potential Regenerative Cell and the technique of Stem cell in situ regeneration are the basic concepts in clinical treatment for wound regenerative treatment (Bianco et al.,2001). The bone marrow provides inflammatory cells and endothelial, mesenchymal progenitor cells to heal the cutaneous wounds. Although the bone marrow contribution of inflammatory cells in the acute response to injury is known, the role of bone marrow derived Mesenchymal Stromal Cells (MSC) in a healed cutaneous wound remain unclear. These cells used for regenerative therapies communicate with the local environments through autocrine and paracrine modalities, thus a favorable optimal environment has been created for healing (Estrada et al., 2009).Hence the analysis of the potential of applying secretome of mesenchymal stromal cell populations from fetal sources for skin wound healing has been carried out to evaluate the potential application of stem cell derivatives for regeneration of tissues in place of cells.

\section{MATERIALS AND METHODS}

All animal procedures were approved under the guidelines of the Institutional Animal ethical Committee, IAEC (Lr. No. 846/DFBS/IAEC/2009 dated 13.04.2010) of Tamil Nadu Veterinary and Animal Sciences University, Tamil Nadu, India. Isolation and culturing of the MSCs were carried out as previously reported (Mangalagowri et al., 2007). Briefly isolated bone marrow cells from fetal bone tissue were selected for CD $45 \%$ CD 14 phenotypes using MACS (magnetic activated cell sorting) and cultured to obtain spindle morphology cells followed by limiting dilution to derive uniform spindle cells. These cells were characterized for surface antigenic phenotypes by immuno-phenotyping. MSCs were grown to $90 \%$ confluency, washed three times with DMEM, and incubated with $4.5 \mathrm{ml}$ of DMEM at $37^{\circ} \mathrm{C}, 5 \% \mathrm{CO}_{2}$ for $42 \mathrm{~h}$. The conditioned medium was collected and centrifuged at $1,000 \mathrm{~g}$ for $10 \mathrm{~min}$ at $48^{\circ} \mathrm{C}$. The resulting supernatant (defined as the stem cell secretome) was aliquoted and frozen at $-80^{\circ} \mathrm{C}$. This was pooled and lyophilized and used for preparation of a gel using paraffin wax as base. Twenty percent ointment of the lyophilized secretome $(\mathrm{w} / \mathrm{w})$ was prepared and used.

Female C57 BL/6J mice of 10 to 12 weeks of age were used. A punch full thickness wound was created on the back of each mouse as described (Frank et al.2000). Briefly, the hair on the backs of the mice was shaved. A full-thickness wound (approximately $5 \mathrm{~mm}$ diameter) was created by excising the skin and the underlying panniculus carnosus. Wound healing activity was assessed in wound models. Wound healing with standard drug (Himax) was done and assessed and the healing pattern was used as base to compare the treatment groups. Two treatment groups containing 6 animals each were used. Control group treated with fibroblast (adult 
sheep skin) secretome was compared with stem cell secretome treatment group. The secretome gel was applied on the wound bed after one hour of wound creation followed by proper removal of dead tissues. At selected time points after wounding, the horizontal $(\mathrm{H})$ and vertical $(\mathrm{V})$ diameters of each wound were measured with a caliper. Percentages of the initial wound area were calculated as: [(V x H) on day n / (V x H) on day 0 x 100]. The hypothesis that MSC treated scars are more pliable was tested objectively by measuring skin elasticity. The collagen content estimation during different days of healing was done by hydroxyproline estimation (Mangalagowri, 2006).

\section{RESULTS}

The collagen estimation during healing days showed a characteristic increase initially followed by decrease in collagen synthesis in stem cell therapy whereas the collagen increase was observed consistently and reduction phase was not observed in fibroblast control (Fig1). On day 3 it was higher than fibroblast treatment,and at peak in around 12 days and consistent upto 21 days of study. Fibroblast cells showed an increasing trend during 15 to 21 days. The tensile strength analysis of the healed tissues in comparison with fibroblast control and uninjured tissue (normal skin) were assessed. The results of this study suggested that the MSC treated sites correlate well with normal skin as measured using Universal Testing machine (INSTRON model 1405) at a cross head speed of $5 \mathrm{~mm} / \mathrm{min}$. This was statistically significant for the parameters (elastic function) and (gross elasticity) as revealed in the Fig.2. The fibroblast control showed that the formation of more fibrous collagen, a factor for scar formation.

\section{DISCUSSION}

The field of wound healing and tissue repair has advanced rapidly in the last decade, with this there is an increasing emphasis on the importance of the functional and cosmetic outcomes following injury. Optimum healing of a cutaneous wound healing requires a well-orchestrated integration of the complex biological and molecular events of cell migration and proliferation, ECM deposition, angiogenesis and remodeling (Falanga et $a l .$, 2005). Understanding this repairing mechanism is important in clinical application of fetal stem cells for regenerative medicine. The murine injury model reported here provides a platform to study the role of fetal stem cells in regeneration of tissues. Animal studies demonstrated that the fetal cells heal cutaneous wounds by reformation of normal tissue architecture without scar formation (Adzick et al., 1992; Mangalagowri et al., 2012) which is supported by the present study. Han et al., (2005) showed that the potential of human bone marrow stromal cells to accelerate wound healing in vitro by measuring the amount of collagen synthesis and the levels of basic fibroblast growth factors and vascular endothelial growth factor. The levels of these growth factors promoting tissue regeneration were shown to be much higher in secretome of the bone marrow stromal cells group compared to the fibroblast group. This factors could have been due to the increased collagen during initial days in stem cell secretome and the same was reported earlier. Ichioka et al., (2005) reported that the addition of bone marrow cells significantly increased the collagen matrix to induce wound healing angiogenesis mainly in the early stage of the repair process. Hence the present healing without scar formation as indicated by the tensile strength similarity of MSC treated skin tissue to normal skin with that of fibroblast cell secretome reveals the potential of fetal cells in regeneration rather than repair of tissue injury.

Also the result of the present study supports the notion that integration of cutting edge technologies in stem cell research would be enhanced by its proteomic analysis, leading to accelerate toward novel stem cell therapies and forms the lead for future research.

\section{CONCLUSION}

This study focuses on the effect of the fetal stem cells on adult wound environment and analyze the tissue repair. The fetus is uniquely capable of healing skin wounds without scar formation and provides a model of ideal tissue repair. Understanding the biology of this process may allow us to modulate wound healing and applying Fetal MSC for tissue repair than adult cells.

\section{ACKNOWLEDGEMENT}

The present findings are part of the research in the DBT, GOI funded project 'Mesenchymal stem cell therapy for induced skin and burn wounds in mice'. The authors place their sincere acknowledgement to the Department of Biotechnology, Government of India (DBT, GOI) New Delhi for funding to carry out the present research and TANUVAS for the facilities provided for the study. 


\section{REFERENCES}

[1]. P Bianco, M Riminucci, S Gronthos and PG Robey, Bonemarrow stromal stem cells: Nature, Biology and potential applications. Stem cells. 19, 2001, 80-92.

[2]. R. Estrada , Na Li, S. Harshini Jin An, Menq-jer lee and Eugenia Wang Secretome from mesenchyaml stem cells induces angiogenesis via Cyr $61 \mathrm{~J}$ Cell Physiol. 219, 2009,563-571.MangalaGowri.,A. Gnanamani and A.Mahalinga Nainar. In vitro isolation and characterization of mesenchymal stem cells from murine bone marrow. Asian J. of Microbial. Biotech. EnvSc., 9, 2007, 929-931.

[3]. Frank,S, H.Kampfer,C.Wetzler. Large induction of the chemotactic cytokine Rantes during cutaneous wound repair. Biochem J.347, 2000, 265-273. Mangalagowri (2006). Ph.D Thesis, isolation and characterization of murine embryonic and bone marrow derived stem cells. Tamilnadu Veterinary and Animal Sciences University.

[4]. V. Falanga. Wound healing and its impairment in the diabetic foot. Lancet, 366:2005,1736- 43.

[5]. NS Adzick, MTLongaker.Scarless fetal healing: Therapeutic implications. Ann Surg 215,1992,3-7.

[6]. A, Mangalagowri, M.Rajasundari, S.Mubeenfarthima.T.M.A.Senthilkumar.Expression of type 1 collagen in undifferentiated MSCs.2012, Dec. IVJ. (in press).

[7]. S.K Han,Potential of human bone marrow stromal cells to accelerate wound healing in vitro. Ann. Plas. Surg. 55, 2005 414-9.

[8]. S. Ichioka, Bone marrow-impregnated collagen matrix for wound healing: experimental evaluation in a microcirculatory model of angiogenesis and clinical experience. Br. J. Plast. Surg. 55, 2005, 414-419.

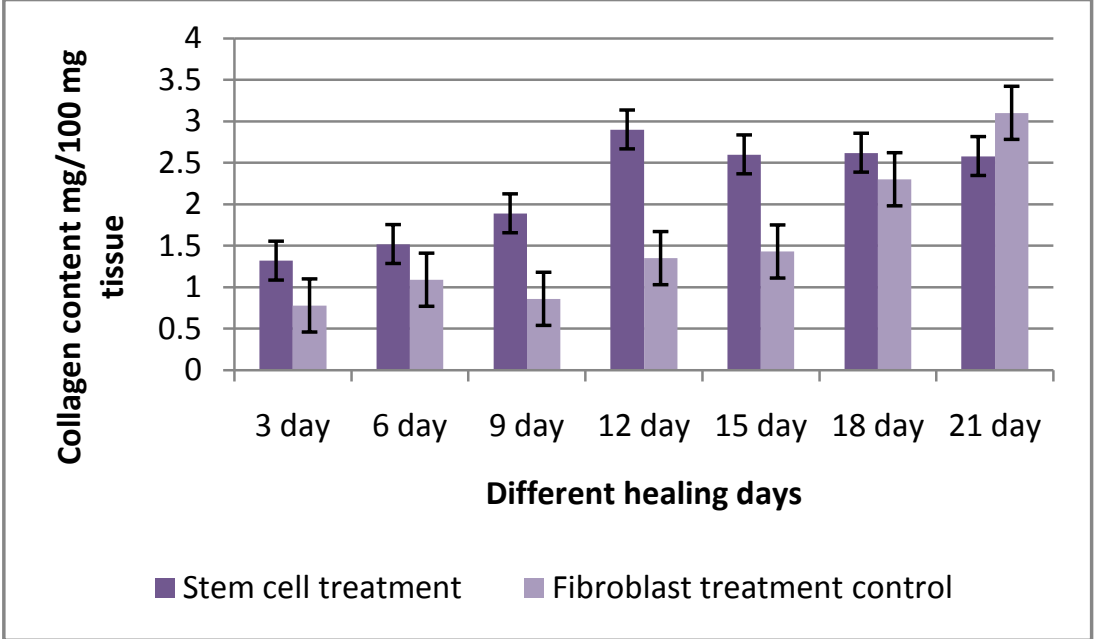

Fig 1: Collagen content estimation in healing granulation tissue

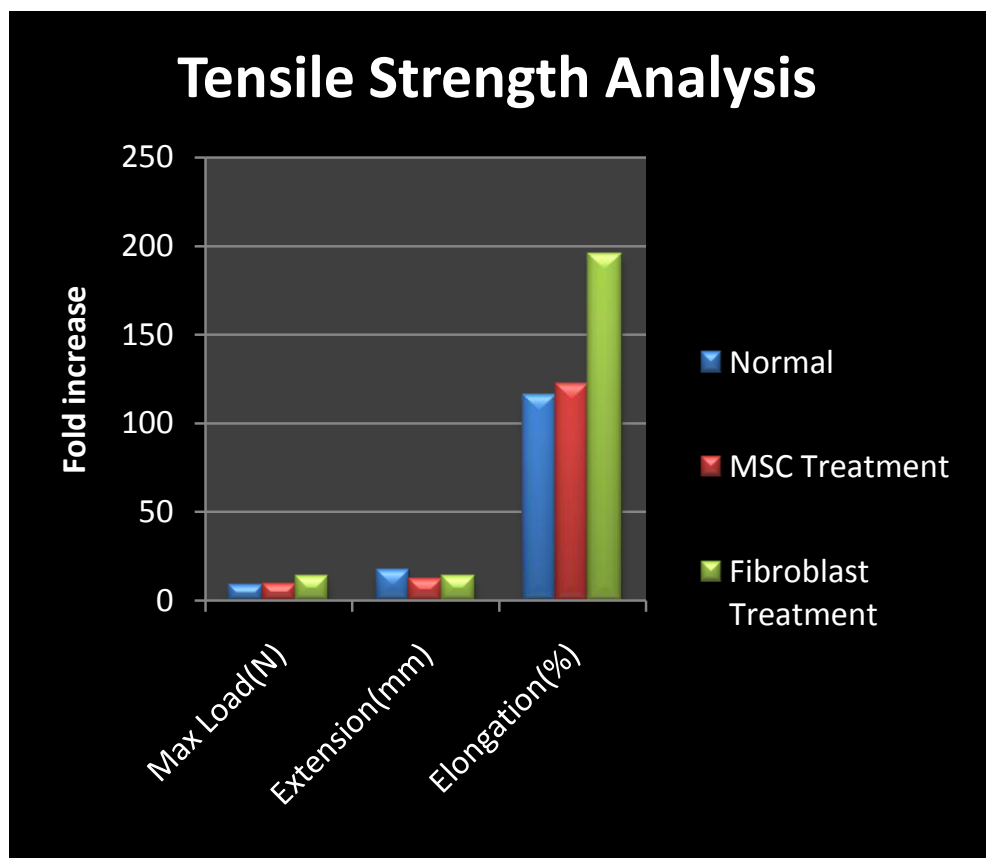

Fig 2: Skin tissue analysis in MSC treatment compared with fibroblast treated and normal tissue 